1 Hacettepe Journal of Mathematics and Statistics

$\bigcap$ Volume $44(2)$ (2015), 261-276

\title{
Near compactness of ditopological texture spaces
}

\author{
Lawrence M. Brown* and Muhammed M. Gohar ${ }^{\dagger}$
}

\begin{abstract}
The authors consider the notions of near compactness, near cocompactness, near stability, near costability and near dicompactness in the setting of ditopological texture spaces. In particular preservation of these properties under surjective $R$-dimaps, co- $R$-dimaps and bi- $R$-dimaps is investigated and non-trivial characterizations of near dicompactness are given which generalize those for dicompactness. The notions of semiregularization, semicoregularization and semibiregularization are defined and used to give generalizations of Mrówka's Theorem for near compactness and near cocompactness, and of Tychonoff's Theorem for near compactness, near cocompactness and near dicompactness. Also, results related to pseudo-open and pseudo-closed sets are presented. Finally, examples are given of co- $T_{1}$ nearly dicompact ditopologies on textures which are not nearly plain and an open question is posed.
\end{abstract}

2000 AMS Classification: 54 A 05, 54 C 10.

Keywords: Texture, Ditopology, Near compactness, Near cocompactness, Near stability, Near costabiliy, Near dicompactness, R-dimaps, co-R-dimaps, Semiregularization, Semicoregularization, Semibiregularization, Tychonoff theorems.

Received 11/01/2013 : Accepted 17/06/2013 Doi : 10.15672/HJMS.2015449101

${ }^{*}$ Hacettepe University, Faculty of Science, Department of Mathematics, Beytepe, Ankara, Turkey.

Email: brown@hacettepe.edu.tr Corresponding Author.

${ }^{\dagger}$ Department of Mathematics, GC University Lahore, Pakistan.

Email: dr.gohar_01@yahoo.com 


\section{Introduction}

The investigation of various types of generalized open set and generalized continuous function is a rich area of research in general topology, and there are interesting applications in various areas, particularly computer science. An early notion in this area is the now well known concept of regular-open set, and its counterpart regular-closed set. Closely related with this notion is the property of near compactness introduced by Singal and Mathur [16, 17], which has been studied by several other authors (see, for example, [11]). Important in this context is the notion of R-map, see [7]. In this paper these and related notions will be studied in the wider context of ditopological texture spaces.

Textures and ditopological texture spaces were first introduced by the first author as a point-based setting for the study of fuzzy sets, and work continues in this direction, see for example [1, 2, 3, 4, 5], and the recent work of Tiryaki [18], Özçă̆ and Brown [15], Yıldız and Brown [22]. On the other hand, textures provide a very convenient setting for the investigation of complement-free concepts in general, so much of the recent work does not involve fuzzy sets explicitly. In particular, the notions of diuniformity and dimetric have been introduced in [14], while a textural analogue of the notion of proximity, called a diextremity, is given in [23].

Compactness in ditopological texture spaces was introduced in [1], its study continued in $[6,20]$, and extended to real compactness in [20] and to strong compactness in [10]. In this paper we place near compactness in a ditopological setting. All the arguments for studying properties related to regular-openness and regular-closedness in the topological setting apply equally well to this case, and since bitopologies and $\mathbb{L}$-topologies, for $\mathbb{L}$ a Hutton algebra, are special cases of ditopologies, the new concepts of near cocompactness, near stability, near costability, and near dicompactness introduced here, may easily be carried over to these settings also.

To complete this introduction we recall various concepts from $[3,4]$ that will be needed later on in this paper.

Ditopological Texture Spaces: If $S$ is a set, a texturing $\mathcal{S}$ of $S$ is a subset of $\mathcal{P}(S)$ which is a point-separating, complete, completely distributive lattice with respect to containment containing $S$ and $\emptyset$, and for which meet coincides with intersection and finite joins with union. The pair $(S, \mathcal{S})$ is then called a texture.

For a texture $(S, \mathcal{S})$, most properties are conveniently defined in terms of the $p$-sets and $q$-sets

$$
P_{s}=\bigcap\{A \in \mathcal{S} \mid s \in A\}, Q_{s}=\bigvee\{A \in \mathcal{S} \mid s \notin A\} .
$$

The following are some basic examples of textures that we will need.

1.1. Examples. (1) If $X$ is a set and $\mathcal{P}(X)$ the powerset of $X$, then $(X, \mathcal{P}(X))$ is the discrete texture on $X$. For $x \in X, P_{x}=\{x\}$ and $Q_{x}=X \backslash\{x\}$.

(2) Setting $\mathbb{I}=[0,1], \mathcal{J}=\{[0, r),[0, r] \mid r \in \mathbb{I}\}$ gives the unit interval texture $(\mathbb{I}, \mathcal{J})$. For $r \in \mathbb{I}, P_{r}=[0, r]$ and $Q_{r}=[0, r)$.

(3) The texture $(L, \mathcal{L})$ is defined by $L=(0,1], \mathcal{L}=\{(0, r] \mid r \in \mathbb{I}\}$. For $r \in L$, $P_{r}=(0, r]=Q_{r}$.

(4) The real texture $(\mathbb{R}, \mathcal{R})$ has $\mathcal{R}=\{(-\infty, r),(-\infty, r] \mid r \in \mathbb{R}\} \cup\{\mathbb{R}, \emptyset\}$. For $r \in \mathbb{R}$, $P_{r}=(-\infty, r]$ and $Q_{r}=(-\infty, r)$.

Since a texturing $\mathcal{S}$ need not be closed under the operation of taking set complement, the notion of topology is replaced by that of dichotomous topology or ditopology, namely a pair $(\tau, \kappa)$ of subsets of $\mathcal{S}$, where the set of open sets $\tau$ satisfies 
(1) $S, \emptyset \in \tau$

(2) $G_{1}, G_{2} \in \tau \Longrightarrow G_{1} \cap G_{2} \in \tau$ and

(3) $G_{i} \in \tau, i \in I \Longrightarrow \bigvee_{i} G_{i} \in \tau$,

and the set of closed sets $\kappa$ satisfies

(1) $S, \emptyset \in \kappa$,

(2) $K_{1}, K_{2} \in \kappa \Longrightarrow K_{1} \cup K_{2} \in \kappa$ and

(3) $K_{i} \in \kappa, i \in I \Longrightarrow \bigcap K_{i} \in \kappa$.

We assume no a priori relation between the open and closed sets. Note that in a ditopology, equal emphasis is placed on the open and closed sets.

For $A \in \mathcal{S}$ we define the closure $[A]$ and the interior $] A[$ of $A$ under $(\tau, \kappa)$ by the equalities

$$
[A]=\bigcap\{K \in \kappa \mid A \subseteq K\} \text { and }] A[=\bigvee\{G \in \tau \mid G \subseteq A\} .
$$

On the other hand, suppose that $(S, \mathcal{S})$ has a complementation $\sigma$, that is an inclusion reversing involution $\sigma: \mathcal{S} \rightarrow \mathcal{S}$. Then if $\tau$ and $\kappa$ are related by $\kappa=\sigma(\tau)$ we say that $(\tau, \kappa)$ is a complemented ditopology on $(S, \mathcal{S}, \sigma)$. In this case we have $\sigma([A])=] \sigma(A)[$ and $\sigma(] A[)=[\sigma(A)]$

We recall the product of textures and of ditopological texture spaces. Let $\left(S_{j}, \mathcal{S}_{j}\right)$, $j \in J$, be textures and $S=\prod_{j \in J} S_{j}$. If $A_{k} \in \mathcal{S}_{k}$ for some $k \in J$ we write

$$
E\left(k, A_{k}\right)=\prod_{j \in J} Y_{j} \text { where } Y_{j}= \begin{cases}A_{j}, & \text { if } j=k \\ S_{j}, & \text { otherwise. }\end{cases}
$$

Then the product texturing $\mathcal{S}=\bigotimes_{j \in J} \mathcal{S}_{j}$ of $S$ consists of arbitrary intersections of elements of the set

$$
\mathcal{E}=\left\{\bigcup_{j \in J} E\left(j, A_{j}\right) \mid A_{j} \in \mathcal{S}_{j} \text { for } j \in J\right\} .
$$

Let $\left(S_{j}, \mathcal{S}_{j}\right), j \in J$ be textures and $(S, \mathcal{S})$ their product. Then for $s=\left(s_{j}\right) \in S$,

$$
P_{s}=\bigcap_{j \in J} E\left(j, P_{s_{j}}\right)=\prod_{j \in J} P_{s_{j}}, \text { and } Q_{s}=\bigcup_{j \in J} E\left(j, Q_{s_{j}}\right) \text {. }
$$

It is easy to verify that for $A_{j} \in \mathcal{S}_{j}, j \in J$ we have $\prod_{j \in J} A_{j} \in \mathcal{S}$.

In case $\left(\tau_{j}, \kappa_{j}\right)$ is a ditopology on $\left(S_{j}, \mathcal{S}_{j}\right), j \in J$, the product ditopology on the product texture $(S, \mathcal{S})$ has subbase $\left\{E(j, G) \mid G \in \tau_{j}, j \in J\right\}$, cosubbase $\gamma=\{E(j, K) \mid$ $\left.K \in \kappa_{j}, j \in J\right\}$.

Let $(S, \mathcal{S}),(T, \mathcal{T})$ be textures. In the following definition we consider the product texture $\mathcal{P}(S) \otimes \mathcal{T}$, and denote by $\bar{P}_{(s, t)}, \bar{Q}_{(s, t)}$, respectively the $p$-sets and $q$-sets for the product texture $(S \times T, \mathcal{P}(S) \otimes \mathcal{T})$.

Direlations: Let $(S, \mathcal{S}),(T, \mathcal{T})$ be textures. Then

(1) $r \in \mathcal{P}(S) \otimes \mathcal{T}$ is called a relation from $(S, \mathcal{S})$ to $(T, \mathcal{T})$ if it satisfies

$R 1 r \nsubseteq \bar{Q}_{(s, t)}, P_{s^{\prime}} \nsubseteq Q_{s} \Longrightarrow r \nsubseteq \bar{Q}_{\left(s^{\prime}, t\right)}$.

$R 2 r \nsubseteq \bar{Q}_{(s, t)} \Longrightarrow \exists s^{\prime} \in S$ such that $P_{s} \nsubseteq Q_{s^{\prime}}$ and $r \nsubseteq \bar{Q}_{\left(s^{\prime}, t\right)}$.

(2) $R \in \mathcal{P}(S) \otimes \mathcal{T}$ is called a corelation from $(S, \mathcal{S})$ to $(T, \mathcal{T})$ if it satisfies

CR1 $\bar{P}_{(s, t)} \nsubseteq \mathbb{R}, P_{s} \nsubseteq Q_{s^{\prime}} \Longrightarrow \bar{P}_{\left(s^{\prime}, t\right)} \not \subset R$.

CR2 $\bar{P}_{(s, t)} \nsubseteq R \Longrightarrow \exists s^{\prime} \in S$ such that $P_{s^{\prime}} \nsubseteq Q_{s}$ and $\bar{P}_{\left(s^{\prime}, t\right)} \nsubseteq R$.

(3) A pair $(r, R)$, where $r$ is a relation and $R$ a corelation from $(S, \mathcal{S})$ to $(T, \mathcal{T})$, is called a direlation from $(S, \mathcal{S})$ to $(T, \mathcal{T})$. 
One of the most useful notions of (ditopological) texture spaces is that of difunction. A difunction is a special type of direlation.

Difunctions: Let $(f, F)$ be a direlation from $(S, \mathcal{S})$ to $(T, \mathcal{T})$. Then $(f, F)$ is called a difunction from $(S, \mathcal{S})$ to $(T, \mathcal{T})$ if it satisfies the following two conditions.

$D F 1$ For $s, s^{\prime} \in S, P_{s} \nsubseteq Q_{s^{\prime}} \Longrightarrow \exists t \in T$ with $f \nsubseteq \bar{Q}_{(s, t)}$ and $\bar{P}_{\left(s^{\prime}, t\right)} \nsubseteq F$.

$D F 2$ For $t, t^{\prime} \in T$ and $s \in S, f \nsubseteq \bar{Q}_{(s, t)}$ and $\bar{P}_{\left(s, t^{\prime}\right)} \not F \Longrightarrow P_{t^{\prime}} \nsubseteq Q_{t}$.

Image and Inverse Image: Let $(f, F):(S, \mathcal{S}) \rightarrow(T, \mathcal{T})$ be a difunction.

(1) For $A \in \mathcal{S}$, the image $f \rightarrow A$ and the co-image $F^{\rightarrow} A$ are defined by

$f^{\rightarrow} A=\bigcap\left\{Q_{t} \mid \forall s, f \nsubseteq \bar{Q}_{(s, t)} \Longrightarrow A \subseteq Q_{s}\right\}$,

$F^{\rightarrow} A=\bigvee\left\{P_{t} \mid \forall s, \bar{P}_{(s, t)} \not \subset F \Longrightarrow P_{s} \subseteq A\right\}$.

(2) For $B \in \mathcal{T}$, the inverse image $f^{\leftarrow} B$ and the inverse co-image $F^{\leftarrow} B$ are defined by

$$
\begin{aligned}
& f^{\leftarrow} B=\bigvee\left\{P_{s} \mid \forall t, f \nsubseteq \bar{Q}_{(s, t)} \Longrightarrow P_{t} \subseteq B\right\}, \\
& F^{\leftarrow} B=\bigcap\left\{Q_{s} \mid \forall t, \bar{P}_{(s, t)} \nsubseteq F \Longrightarrow B \subseteq Q_{t}\right\} .
\end{aligned}
$$

For a difunction, the inverse image and the inverse co-image are equal, but the image and co-image are generally different. A difunction is called surjective if it satisfies

SUR. For $t, t^{\prime} \in T, P_{t} \nsubseteq Q_{t^{\prime}} \Longrightarrow \exists s \in S$ with $f \nsubseteq \bar{Q}_{\left(s, t^{\prime}\right)}$ and $\bar{P}_{(s, t)} \nsubseteq F$.

Bicontinuity: The difunction $\left.(f, F):\left(S, \mathcal{S}, \tau_{S}, \kappa_{S}\right)\right) \rightarrow\left(T, \mathcal{T}, \tau_{T}, \kappa_{T}\right)$ is called continuous if $B \in \tau_{T} \Longrightarrow F^{\leftarrow} B \in \tau_{S}$, cocontinuous if $B \in \kappa_{T} \Longrightarrow f^{\leftarrow} B \in \kappa_{S}$, and bicontinuous if it is both continuous and cocontinuous.

On the other hand $(f, F)$ is open (co-open) if $A \in \tau_{S} \Longrightarrow f^{\rightarrow} A \in \tau_{T}\left(F^{\rightarrow} A \in \tau_{T}\right)$. Also, $(f, F)$ is closed (coclosed) if $A \in \kappa_{S} \Longrightarrow f \rightarrow A \in \kappa_{T}\left(F^{\rightarrow} A \in \kappa_{T}\right)$.

If $\left(S_{j}, \mathcal{S}_{j}, \tau_{j}, \kappa_{j}\right), j \in J$, are ditopological texture spaces and $(S, \mathcal{S}, \tau, \kappa)$ their product, the projection difunctions $\left(\pi_{j}, \Pi_{j}\right):(S, \mathcal{S}, \tau, \kappa) \rightarrow\left(S_{j}, \mathcal{S}_{j}, \tau_{j}, \kappa_{j}\right)$ are important examples of surjective bicontinouous difunctions that satisfy $\pi_{j}^{\leftarrow} B=E(j, B)=\Pi_{j}^{\leftarrow} B$ for all $B \in \mathcal{S}_{j}$ (see [4] and [6]).

Separation Axioms A comprehensive treatment is given in [5], and the definitions will not be repeated here.

The layout of the paper is as follows. In Section 2 the concepts of near compactness and near cocompactness are given in the setting of ditopological texture spaces, while Section 3 deals with near stability and near costability, which are analogues for near compactness of the notion of stability in bitopological spaces introduced by Ralph Kopperman [12]. Section 4 deals with the preservation of these properties under surjective continuous difunctions via the notions of R-dimap and co-R-dimap, while Section 5 combines them in the notion of near dicompactness and provides important characterizations of this important concept. Finally Section 6 introduces the semibiregularization of a ditopological texture space, gives generalizations of the Mrówka characterization of compactness for near compactness and near cocompactness, discuses characterizations of near compact and near cocompact sets in near compact and near cocompact spaces using the notions of pseudo open and pseudo closed set, and gives versions of the Tychonoff product theorem for near compactness and near cocompactness. Here also we look at near dicompactness in the presence of point separation axioms.

This work includes results, many reformulated, from an unpublished section of the $\mathrm{PhD}$ thesis of the second author [9] together with interesting new material.

For terms from lattice theory not defined here the reader is referred to [8]. 


\section{Near compactness and near cocompactness}

The notion of nearly compact topological space was introduced by Singal and Mathur $[16,17]$, and has been studied by several other authors (see, for example, [11]). The definition of near compactness requires the concepts of regular-open and regular-closed sets in a topological space. We recall the definitions below:

2.1. Definition. Let $(X, \mathcal{T})$ be a topological space.

(1) If $A \subseteq X$ satisfies $A=\operatorname{int}(\operatorname{cl}(A))$ then $A$ is called a regular-open set.

(2) If $B \subseteq X$ satisfies $B=\operatorname{cl}(\operatorname{int}(B)))$ then $B$ is called a regular-closed set.

2.2. Examples. (1) Every regular-open set is open and every regular-closed set is closed. Moreover the complement of a regular-closed set is regular-open and the complement of a regular-open set is regular-closed.

(2) Let $X=\{a, b\}$ and let the topology on $X$ be $\mathcal{T}=\{\emptyset, X,\{a\}\}$. Here $\{b\}$ is a closed set but $\operatorname{cl}(\operatorname{int}(\{b\}))=\emptyset$, so $\{b\}$ is not regular-closed.

2.3. Lemma. Let $(X, \mathcal{T})$ be a topological space, $A, B \subseteq X$. Then $\operatorname{int}(\operatorname{cl}(A))$ is a regularopen set and $\operatorname{cl}(\operatorname{int}(B))$ a regular-closed set

In case of ditopological texture spaces we may give corresponding definitions, as follows.

2.4. Definition. Let $(\tau, \kappa)$ be a ditopology on the texture $(S, \mathcal{S})$.

(1) An element $A \in \mathcal{S}$ will be called regular-open if $][A][=A$.

(2) An element $B \in \mathcal{S}$ will be called regular-closed if [] $A[]=A$

It is clear that sets of the form $][A][, A \in \mathcal{S}$, are regular-open and those of the form [] $A[], A \in \mathcal{S}$ are regular-closed. In general there is no relation between regular-open and regular-closed sets in a ditopological texture space, but for complemented ditopological texture spaces we do have the following result.

2.5. Proposition. Let $(S, \mathcal{S}, \sigma, \tau, \kappa)$ be a complemented ditopological texture space and $A \in \mathcal{S}$. Then

(1) $A \in \mathcal{S}$ is regular-open if and only if $\sigma(A)$ is regular-closed.

(2) $A \in \mathcal{S}$ is regular-closed if and only if $\sigma(A)$ is regular-open.

Proof. Straightforward.

Now let us recall the definition of nearly compact topological space.

2.6. Definition. A topological space $(X, \mathcal{T})$ is said to be nearly compact if every open cover $\left\{U_{i} \mid i \in I\right\}$ of $X$ admits a finite subfamily such that $X=\bigcup_{i=1}^{n} \operatorname{int}\left(\operatorname{cl}\left(U_{i}\right)\right)$. That is, $X$ is nearly compact if and only if every regular-open cover of $X$ has a finite subcover.

The set $\mathbb{R}$ with its usual topology and $\mathbb{R}$ with the discrete topology are not nearly compact. However the indiscrete topology and the left ray topology $\{(-\infty, r) \mid r \in$ $\mathbb{R}\} \cup\{\mathbb{R}, \emptyset\}$ on $\mathbb{R}$ are examples of nearly compact topological spaces.

In the case of ditopological texture spaces we may give a corresponding definition of near compactness, and a dual notion of near cocompactness, as follows.

2.7. Definition. Let $(S, \mathcal{S}, \tau, \kappa)$ be a ditopological texture space and $A \in \mathcal{S}$.

(1) $A$ will be called nearly compact in $S$ if whenever $A \subseteq \bigvee_{i \in I} G_{i}, G_{i} \in \tau$, there exists $I^{\prime} \subseteq I$ finite, so that $\left.A \subseteq \bigcup_{i \in I^{\prime}}\right]\left[G_{i}\right][$. The ditopological texture space $(S, \mathcal{S}, \tau, \kappa)$ will be called nearly compact if $S$ is nearly compact in $S$. 
(2) $A$ will be called nearly cocompact in $S$ if whenever $\bigcap_{i \in I} F_{i} \subseteq A, F_{i} \in \kappa$, there exists $I^{\prime} \subseteq I$ finite, so that $\bigcap_{i \in I^{\prime}}[] F_{i}[] \subseteq A$. The ditopological texture space $(S, \mathcal{S}, \tau, \kappa)$ will be called nearly cocompact if $\emptyset$ is nearly cocompact in $S$.

As in the topological case we have the following characterizations.

2.8. Proposition. Let $(S, \mathcal{S}, \tau, \kappa)$ be a ditopological texture space and $A \in \mathcal{S}$.

(1) $A$ is nearly compact in $S$ if and only if every cover of $A$ by regular-open sets in $\mathcal{S}$ has a finite subcover.

(2) $A$ is nearly cocompact in $S$ if every cocover of $A$ by regular-open sets in $\mathcal{S}$ has a finite sub-cocover.

Proof. (1). Let $A$ be nearly compact in $S$ and suppose $G_{i}, i \in I$ is cover of $A$ by regularopen sets. Since in particular $G_{i} \in \tau$, we have $I^{\prime}$ finite with $\left.A \subseteq \bigcup_{i \in I^{\prime}}\right]\left[G_{i}\right]\left[=\bigcup_{i \in I^{\prime}} G_{i}\right.$ since the sets $G_{i}$ are regular-open. Hence $\left\{G_{i} \mid i \in I\right\}$ has a finite subcover.

Conversely, suppose every regular-open cover of $A$ has a finite subcover, and let $\left\{G_{i} \mid\right.$ $i \in I\}$ be an open cover of $A$. For each $\left.i \in I, G_{i} \subseteq\right]\left[G_{i}\right]\left[\right.$, so \{]$\left[G_{i}\right][\mid i \in I\}$ is a cover of $A$ by regular-open sets. By hypothesis there exists $I^{\prime} \subseteq I$ finite with $\left.A \subseteq \bigcup_{i \in I^{\prime}}\right]\left[G_{i}\right][$, which shows that $A$ is nearly compact.

(2). The proof is dual to the above, and is therefore omitted.

2.9. Corollary. Let $(S, \mathcal{S}, \tau, \kappa)$ be a ditopological texture space and $A \in \mathcal{S}$.

(1) If $A$ is compact in $S$ it is nearly compact in $S$.

(2) If $A$ is cocompact in $S$ it is nearly cocompact in $S$.

Proof. Clear from Proposition 2.8 since every regular-open set is open and every regularclosed set is closed.

The following examples show that the converse of Corollary 2.9 is false. Also, in general, the properties of near compactness and near cocompactness are independent of one another.

2.10. Examples. Consider the texture $(L, \mathcal{L})$ as in Examples 1.1.

(1) Let $\tau=\mathcal{L}$ and $\kappa=\{L, \emptyset\}$. If we take $r$ with $0<r<1$ and set $A=(0, r]$, then $[A]=L$ and so $][A][=] L[\neq A$, from which we see that $A$ is not regular-open. It follows that the only regular-open sets are $\emptyset$ and $L$, so $(\tau, \kappa)$ is nearly compact because any regular-open cover of $L$ is finite. On the other hand $(\tau, \kappa)$ is not compact by [6, Example $2.2(2)]$.

(2) In just the same way, $\tau=\{\emptyset, L\}$ and $\kappa=\mathcal{L}$ define a ditopology which is nearly cocompact but not cocompact.

(3) Take $\tau=\{(0, r] \mid 0 \leq r \leq 1 / 2\} \cup\{L\}$ and $\kappa=\mathcal{L}$. This ditopology $(\tau, \kappa)$ is not nearly co-compact, since for example $\mathcal{C}=\{(0,1 / n] \mid n=1,2 \ldots\}$ is a regularclosed co-cover of $\emptyset$ which has no finite sub co-cover. However $(\tau, \kappa)$ is nearly compact because it is compact.

(4) In just the same way $\tau=\mathcal{L}$ and $\kappa=\{(0, r] \mid 1 / 2 \leq r \leq 1\} \cup\{\emptyset\}$ defines a ditopology which is nearly co-compact but not nearly compact.

For complemented ditopologies, however, near compactness and near cocompactness are equivalent.

2.11. Proposition. Let $(S, \mathcal{S}, \sigma, \tau, \kappa)$ be a complemented ditopological texture space. Then $(\tau, \kappa)$ is nearly compact if and only if $(\tau, \kappa)$ is nearly cocompact.

Proof. Straightforward. 


\section{Near stability and near costability}

We now wish to generalize the notions of stability and costability. The following definition would appear to be appropriate.

3.1. Definition. Let $(\tau, \kappa)$ be a ditopology on the texture space $(S, \mathcal{S})$.

(1) $(\tau, \kappa)$ will be called nearly stable if every set $F \in \mathcal{S} \backslash\{S\}$ of the form $F=$ $\bigcap_{\alpha \in A} K_{\alpha}, K_{\alpha}$ regular-closed for each $\alpha \in A$, is nearly compact in $S$.

(2) $(\tau, \kappa)$ will be called nearly costable if every set $G \in \mathcal{S} \backslash \emptyset$ of the form $G=\bigvee_{\alpha \in A} G_{\alpha}$, $G_{\alpha}$ regular-open for each $\alpha \in A$, is nearly cocompact in $S$.

3.2. Example. Consider the texture $(L, \mathcal{L})$, as in previous examples,

(1) Take $\tau=\mathcal{L}$ and $\kappa=\{\emptyset,(0,1 / 2], L\}$. As we have noted earlier, $(\tau, \kappa)$ is not stable because $(0,1 / 2]$ is a closed set which is not compact. On the other hand the only regular-open sets in this space are $\emptyset,(0,1 / 2]$ and $L$, so it is nearly stable.

(2) Dually, $(\tau, \kappa)$ defined by $\tau=\{\emptyset,(0,1 / 2], L\}$ and $\kappa=\mathcal{L}$ is nearly costable but not costable.

(3) Let $\tau=\kappa=\{(0,1 / 2-1 / n] \mid n \geq 2\} \cup\{(0,1 / 2], L\}$. The regular-closed set $(0,1 / 2]$ is not nearly compact since $\{(0,1 / 2-1 / n] \mid n \geq 2\}$ is a cover by regularopen sets which has no finite subcover, so $(\tau, \kappa)$ is not nearly stable. On the other hand it is clearly nearly costable.

(4) Dually, $(\tau, \kappa)$ defined by $\tau=\kappa=\{(0,1 / 2+1 / n] \mid n \geq 2\} \cup\{\emptyset,(0,1 / 2]\}$ is nearly stable but not nearly costable.

The last two examples show that in general near stability and near costability are independent of one another. However for complemented ditopological texture spaces these concepts are equivalent, as we now show.

3.3. Proposition. Let $(S, \mathcal{S}, \sigma)$ be a texture with complementation $\sigma$ and $(\tau, \kappa)$ a complemented ditopology on $(S, \mathcal{S}, \sigma)$. Then $(\tau, \kappa)$ is nearly stable if and only if $(\tau, \kappa)$ is nearly costable.

Proof. Suppose that $(\tau, \kappa)$ is nearly stable. Let $\emptyset \neq G=\bigvee_{\alpha \in A} G_{\alpha}$ with $G_{\alpha}$ regular-open for each $\alpha$. Also, let $\mathcal{D}$ be a regular-closed cocover of $G$, i.e.,

$$
\bigcap \mathcal{D} \subseteq G=\bigvee G_{\alpha}
$$

If we let $K_{\alpha}=\sigma\left(G_{\alpha}\right)$ then $K_{\alpha}$ is regular-closed and $K=\sigma(G)=\sigma\left(\bigvee G_{\alpha}\right)=\bigcap \sigma\left(G_{\alpha}\right)=$ $\bigcap_{\alpha \in A} K_{\alpha}$ satisfies $K \neq S$. Also,

$$
\mathcal{C}=\{\sigma(F) \mid F \in \mathcal{D}\}
$$

is a cover of $K$ by regular-open sets. By hypothesis $K$ is nearly compact so there exists $\alpha_{1}, \ldots \alpha_{n} \in A$ such that

$$
K \subseteq \sigma\left(F_{\alpha_{1}}\right) \cup \sigma\left(F_{\alpha_{2}}\right) \cup \ldots \cup \sigma\left(F_{\alpha_{n}}\right)=\sigma\left(F_{\alpha_{1}} \cap F_{\alpha_{2}} \cap \ldots \cap F_{\alpha_{n}}\right),
$$

so $F_{\alpha_{1}} \cap F_{\alpha_{2}} \cap \ldots \cap F_{\alpha_{n}} \subseteq \sigma(K)=G$, which proves that $G$ is nearly cocompact. Hence, $(\tau, \kappa)$ is nearly costable.

Conversely, if $(\tau, \kappa)$ is nearly costable it is nearly stable. The proof is dual to the above, and is omitted.

\section{4. $R$-dimaps and co- $R$-dimaps}

Now let us consider the preservation of the above properties under surjective difunctions. It is known [7] that near compactness of topological spaces is preserved under $R$-maps, so we begin by recalling the definition of $R$-map. 
4.1. Definition. Let $\left(X_{1}, \mathcal{T}_{1}\right),\left(X_{2}, \mathcal{T}_{2}\right)$ be topological spaces and $f: X_{1} \rightarrow X_{2}$ a function. If for every regular-open set $B \subseteq X_{2}$ the set $f^{-1}(B)$ is regular-open in $X_{1}$ the function $f$ is called an $R$-map.

We make corresponding definitions for ditopological texture spaces.

4.2. Definition. Let $\left(S_{1}, \mathcal{S}_{1}, \tau_{1}, \kappa_{1}\right),\left(S_{2}, \mathcal{S}_{2}, \tau_{2}, \kappa_{2}\right)$ be ditopological texture spaces and $(f, F):\left(S_{1}, \mathcal{S}_{1}\right) \rightarrow\left(S_{2}, \mathcal{S}_{2}\right)$ a difunction.

(1) $(f, F)$ will be said to be an $R$-dimap if for every regular-open set $B \in \mathcal{S}_{2}$ the set $F^{\leftarrow}(B) \in \mathcal{S}_{1}$ is regular-open .

(2) $(f, F)$ will be said to be a co-R-dimap if for every regular-closed set $B \in \mathcal{S}_{2}$ the set $f^{\leftarrow}(B) \in \mathcal{S}_{1}$ is regular-closed.

(3) $(f, F)$ will be said to be a bi-R-dimap if it is an $R$-dimap and a co- $R$-dimap.

Now we may state and prove the following theorems, which generalize the topological case.

4.3. Theorem. Let $(f, F):\left(S_{1}, \mathcal{S}_{1}, \tau_{1}, \kappa_{1}\right) \rightarrow\left(S_{2}, \mathcal{S}_{2}, \tau_{2}, \kappa_{2}\right)$ be a surjective $R$-dimap. If $\left(S_{1}, \mathcal{S}_{1}, \tau_{1}, \kappa_{1}\right)$ is nearly compact then $\left(S_{2}, \mathcal{S}_{2}, \tau_{2}, \kappa_{2}\right)$ is nearly compact.

Proof. Assume $S_{2}=\bigvee_{\alpha \in A} G_{\alpha}$, where the sets $G_{\alpha} \in \mathcal{S}_{2}$ are regular-open . Since $(f, F)$ is a difunction we have $F^{\leftarrow}\left(S_{2}\right)=S_{1}$ by [3, Proposition $\left.2.28(1 \mathrm{c})\right]$, so

$$
S_{1}=F^{\leftarrow}\left(S_{2}\right)=F^{\leftarrow}\left(\bigvee_{\alpha \in A} G_{\alpha}\right)=\bigvee_{\alpha \in A} F^{\leftarrow}\left(G_{\alpha}\right),
$$

by [3, Corollary $2.12(2)]$. Also, $F^{\leftarrow}\left(G_{\alpha}\right)$ is regular-open for each $\alpha \in A$ since $(f, F)$ is a $R$-bimap. Hence, by the near compactness of $\left(S_{1}, \mathcal{S}_{1}, \tau_{1}, \kappa_{1}\right)$ there exists $A^{\prime} \subseteq A$ finite such that $S_{1}=\bigcup_{\alpha \in A^{\prime}} F^{\leftarrow}\left(G_{\alpha}\right)$. Hence

$$
f^{\leftarrow}\left(S_{2}\right)=S_{1}=\bigcup_{\alpha \in A^{\prime}} F^{\leftarrow}\left(G_{\alpha}\right)=F^{\leftarrow}\left(\bigcup_{\alpha \in A^{\prime}} G_{\alpha}\right) .
$$

Since $(f, F)$ is surjective $f^{\leftarrow}\left(S_{2}\right) \subseteq F^{\leftarrow}\left(\bigcup_{\alpha \in A^{\prime}} G_{\alpha}\right)$ implies that $S_{2} \subseteq \bigcup_{\alpha \in A^{\prime}} G_{\alpha}$ by [3, Corollary 2.33(1ii)]. Therefore, $S_{2}=\bigcup_{\alpha \in A^{\prime}} G_{\alpha}$, and so $\left(S_{2}, \mathcal{S}_{2}, \tau_{2}, \kappa_{2}\right)$ is nearly compact.

As expected, we have a dual theorem for near cocompactness.

4.4. Theorem. Let $(f, F):\left(S_{1}, \mathcal{S}_{1}, \tau_{1}, \kappa_{1}\right) \rightarrow\left(S_{2}, \mathcal{S}_{2}, \tau_{2}, \kappa_{2}\right)$ be a surjective co $R$-dimap. If $\left(S_{1}, \mathcal{S}_{1}, \tau_{1}, \kappa_{1}\right)$ is nearly cocompact then $\left(S_{2}, \mathcal{S}_{2}, \tau_{2}, \kappa_{2}\right)$ is nearly cocompact.

Proof. This is dual to the proof of Theorem 4.3, and is omitted.

Next let us investigate the preservation of near stability and near costability under surjective difunctions.

4.5. Theorem. Let $\left(S_{1}, \mathcal{S}_{1} \tau_{1}, \kappa_{1}\right),\left(S_{2}, \mathcal{S}_{2}, \tau_{2}, \kappa_{2}\right)$ be ditopological texture spaces with $\left(\tau_{1}, \kappa_{1}\right)$ nearly stable, and $(f, F):\left(S_{1}, \mathcal{S}_{1}, \tau_{1}, \kappa_{1}\right) \rightarrow\left(S_{2}, \mathcal{S}_{2}, \tau_{2}, \kappa_{2}\right)$ a surjective bi- $R$ dimap. Then $\left(\tau_{2}, \kappa_{2}\right)$ is nearly stable.

Proof. Take $K \in S_{2} \backslash\left\{S_{2}\right\}$ of the form $K=\bigcap_{\alpha \in A} K_{\alpha}$ with $K_{\alpha}$ regular-closed for each $\alpha \in A$. We must prove that $K$ is nearly compact for the ditopology $\left(\tau_{2}, \kappa_{2}\right)$ so take regular-open sets $G_{i}, i \in I$, satisfying

$$
K \subseteq \bigvee\left\{G_{i} \mid i \in I\right\}
$$

Since $(f, F)$ is a co- $R$-dimap, $f^{\leftarrow}(K)=f^{\leftarrow}\left(\bigcap_{\alpha \in A} K_{\alpha}\right)=\bigcap_{\alpha \in A} f^{\leftarrow}\left(K_{\alpha}\right)$ is an intersection of regular-closed sets in $\left(S_{1}, \mathcal{S}_{1}\right)$. As in the proof of [6, Theorem 3.17], $f^{\leftarrow}(K) \neq S_{1}$. Hence $f^{\leftarrow}(K)$ is nearly compact in $\left(S_{1}, \mathcal{S}_{1}, \tau_{1}, \kappa_{1}\right)$, since this space is nearly stable. 
From (4.1) we have

$$
f^{\leftarrow}(K) \subseteq f^{\leftarrow}\left(\bigvee\left\{G_{i} \mid i \in I\right\}\right)=F^{\leftarrow}\left(\bigvee\left\{G_{i} \mid i \in I\right\}\right)=\bigvee\left\{F^{\leftarrow}\left(G_{i}\right) \mid i \in I\right\}
$$

by [3, Corollary $2.12(2)]$, and $F^{\leftarrow}\left(G_{i}\right)$ is regular-open since $(f, F)$ is an $R$-dimap. By near compactness we have $i_{1}, i_{2}, \ldots, i_{n} \in I$ with

$$
\begin{aligned}
f^{\leftarrow}(K) & \subseteq \bigcup_{k=1}^{n} F^{\leftarrow}\left(G_{i_{k}}\right) \\
& =F^{\leftarrow}\left(\bigcup_{k=1}^{n} G_{i_{k}}\right)=f^{\leftarrow}\left(\bigcup_{k=1}^{n} G_{i_{k}}\right) .
\end{aligned}
$$

Since $(f, F)$ is surjective we have

$$
K=F\left(f^{\leftarrow}(K)\right) \subseteq F\left(f^{\leftarrow}\left(\bigcup_{k=1}^{n} G_{i_{k}}\right)\right)=\bigcup_{k=1}^{n} G_{i_{k}} .
$$

This shows that $K$ is nearly compact and completes the proof that $\left(\tau_{2}, \kappa_{2}\right)$ is nearly stable.

4.6. Theorem. Let $\left(S_{1}, \mathcal{S}_{1} \tau_{1}, \kappa_{1}\right),\left(S_{2}, \mathcal{S}_{2}, \tau_{2}, \kappa_{2}\right)$ be ditopological texture spaces with $\left(\tau_{1}, \kappa_{1}\right)$ nearly costable, and $(f, F):\left(S_{1}, \mathcal{S}_{1}, \tau_{1}, \kappa_{1}\right) \rightarrow\left(S_{2}, \mathcal{S}_{2}, \tau_{2}, \kappa_{2}\right)$ a surjective bi$R$-dimap. Then $\left(\tau_{2}, \kappa_{2}\right)$ is nearly costable.

Proof. The proof is dual to that of Theorem 4.5, and is omitted.

\section{Near dicompactness}

The notion of dicompactness may be generalized for near compactness as follows.

5.1. Definition. A ditopological texture space will be called nearly dicompact if it is nearly compact, nearly cocompact, nearly stable and nearly costable.

As a consequence of Theorems 4.3, 4.4, 4.5 and 4.6 we may state the following:

5.2. Theorem. Near dicompactness is preserved under a surjective bi-R-dimap.

To give non-trivial characterizations of near dicompactness analogous to those for dicompact ditopological texture spaces we require the following definitions that are adapted from $[1,6]$.

5.3. Definition. Let $(\tau, \kappa)$ be a ditopology on $(S, \mathcal{S})$.

(1) A set $\mathcal{D} \subseteq \mathcal{S} \times \mathcal{S}$ is called a difamily on $(S, \mathcal{S})$. A difamily $\mathcal{D}$ is called regular-closed, co-regular-open if $A \mathcal{D} B$ implies $A$ is regular-closed and $B$ is regular-open, while it is called regular-open, co-regular-closed if $A$ is regular-open and $B$ is regularclosed.

(2) A difamily $\mathcal{D}$ has the finite exclusion property (fep) if whenever $\left(F_{i}, G_{i}\right) \in \mathcal{D}$, $i=1,2, \ldots, n$ we have $\bigcap_{i=1}^{n} F_{i} \nsubseteq \bigcup_{i=1}^{n} G_{i}$.

(3) A regular-closed, co-regular-open difamily $\mathcal{D}$ with $\bigcap\{F \mid F \in \operatorname{dom} \mathcal{D}\} \nsubseteq \bigvee\{G \mid$ $G \in \operatorname{ran} \mathcal{D}\}$ is said to be bound in $(S, \mathcal{S}, \tau, \kappa)$.

(4) A difamily $\mathcal{D}=\left\{\left(G_{i}, F_{i}\right) \mid i \in I\right\}$ is called a dicover of $(S, \mathcal{S})$ if for all partitions $I_{1}, I_{2}$ of $I$ (including the trivial partitions) we have

$\bigcap_{i \in I_{1}} F_{i} \subseteq \bigvee_{i \in I_{2}} G_{i}$

(5) A difamily $\mathcal{D}$ is called finite (co-finite) if dom $\mathcal{D}$ (resp. ran $\mathcal{D})$ is finite. 
5.4. Theorem. The following are equivalent for a ditopological texture space $(S, \mathcal{S}, \tau, \kappa)$.

(1) $(S, \mathcal{S}, \tau, \kappa)$ is nearly dicompact.

(2) Every regular-closed, co-regular-open difamily with the finite exclusion property is bound.

(3) Every regular-open, co-regular-closed dicover has a sub-dicover which is finite and co-finite.

Proof. (1) $\Longrightarrow(2)$. Suppose that (1) holds, but that we have a regular-closed, co-regularopen difamily $\mathcal{B}=\left\{\left(F_{i}, G_{i}\right) \mid i \in I\right\}$ with the fep, which is not bound in $(S, \mathcal{S}, \tau, \kappa)$. Let $F=\bigcap_{i \in I} F_{i}$. Then $F$ is an intersection of regular-closed sets, and $F \subseteq \bigvee_{i \in I} G_{i}$ since $\mathcal{B}$ is not bound. According as $F \neq S$ or $F=S$ we may use near stability or near compactness, respectively, to show the existence of a finite subset $J_{1}$ of $I$ with $F \subseteq \bigcup_{j \in J_{1}} G_{j}$. Now let $G=\bigvee_{j \in J_{1}} G_{j}$. Then $G$ is a join of regular-open sets and $\bigcap_{i \in I} F_{i} \subseteq G$. Hence, according as $G \neq \emptyset$ or $G=\emptyset$, we may use near costability or near cocompactness, respectively, to show that $\bigcap_{j \in J_{2}} F_{j} \subseteq G$ for some finite subset $J_{2}$ of $I$. Since now $\bigcap_{j \in J_{1} \cup J_{2}} F_{j} \subseteq \bigcup_{j \in J_{1} \cup J_{2}} G_{j}$ we have a contradiction to the fact that $\mathcal{B}$ has the fep.

$(2) \Longrightarrow(3)$. Suppose that $\mathcal{C}=\left\{\left(G_{i}, F_{i}\right) \mid i \in I\right\}$ is a regular-open, co-regular-closed dicover with no finite, co-finite sub-dicover. As in the proof of $[1$, Theorem 3.5] we consider the set $\mathcal{F}$ of functions $f$ satisfying

(a) $\operatorname{dom} f$ is a set of finite subsets of $I$.

(b) $\forall J \in \operatorname{dom} f, f(J)=\left(f_{1}(J), f_{2}(J)\right) \in \mathcal{P}_{J}^{\star \star}$.

(c) $J_{1}, \ldots, J_{n} \in \operatorname{dom} f \Longrightarrow J_{1} \cup \ldots \cup J_{n} \in \operatorname{dom} f$.

(d) $J, K \in \operatorname{dom} f, J \subseteq K \Longrightarrow f_{l}(J)=J \cap f_{l}(K), l=1,2$.

Here

$$
\begin{aligned}
\mathcal{P}_{J}^{\star \star}=\left\{\left(J_{1}, J_{2}\right) \in \mathcal{P}_{J}^{\star} \mid \forall K \text { finite, } J \subseteq K \subseteq I,\right. & \exists\left(K_{1}, K_{2}\right) \in \mathcal{P}_{K}^{\star} \\
& \text { with } \left.J \cap K_{l}=J_{l}, l=1,2\right\} .
\end{aligned}
$$

where

$$
\begin{aligned}
& \mathcal{P}_{J}=\left\{\left(J_{1}, J_{2}\right) \mid J=J_{1} \sqcup J_{2}\right\}, \text { and } \\
& \mathcal{P}_{J}^{\star}=\left\{\left(J_{1}, J_{2}\right) \in \mathcal{P}_{J} \mid \bigcap_{j \in J_{1}} F_{j} \nsubseteq \bigvee_{j \in J_{2}} G_{j}\right\},
\end{aligned}
$$

and $J=J_{1} \sqcup J_{2}$ denotes that $\left\{J_{1}, J_{2}\right\}$ is a partition of $J$. Just as in the proof of [1, Theorem 3.5] we may establish that $\mathcal{F}$ contains an element $g$ satisfying $\bigcup \operatorname{dom} g=I$.

Now consider the family $\mathcal{B}=\left\{\left(F_{j}, G_{k}\right) \mid j \in g_{1}(J), k \in g_{2}(J), J \in \operatorname{dom} g\right\}$. It is easy to show that $\mathcal{B}$ has the fep. Also $F_{j}$ is regular-closed and $G_{k}$ is regular-open, so by (2) we have

$$
\bigcap_{J \in \operatorname{dom} g}\left(\bigcap_{j \in g_{1}(J)} F_{j}\right) \not \underset{J \in \operatorname{dom} g}{\bigvee}\left(\bigcup_{j \in g_{2}(J)} G_{j}\right)
$$

Let $I_{1}=\bigcup\left\{g_{1}(J) \mid J \in \operatorname{dom} g\right\}, I_{2}=I \backslash I_{1}$. Then $\left(I_{1}, I_{2}\right)$ is a partition of $I$, and $I_{2} \subseteq \bigcup\left\{g_{2}(J) \mid J \in \operatorname{dom} g\right\}$. This gives us

$$
\bigcap_{J \in \operatorname{dom} g}\left(\bigcap_{j \in g_{1}(J)} F_{j}\right)=\bigcap_{i \in I_{1}} F_{i} \subseteq \bigvee_{i \in I_{2}} G_{i} \subseteq \bigvee_{J \in \operatorname{dom} g}\left(\bigcup_{j \in g_{2}(J)} G_{j}\right),
$$

which is a contradiction.

$(3) \Longrightarrow(1)$. First take regular-open sets $G_{i}, i \in I$, with $S=\bigvee_{i \in I} G_{i}$. For $i \in I$ let $F_{i}=\emptyset$. Then $\mathcal{C}=\left\{\left(G_{i}, F_{i}\right) \mid i \in I\right\}$ is a regular-open, co-regular-closed dicover, so has 
a finite, co-finite sub-dicover $\left\{\left(G_{j}, F_{j}\right) \mid j \in J\right\}$. For the partition $J_{1}=\emptyset, J_{2}=J$ of $J$,

$$
S=\bigcap_{j \in J_{1}} F_{j} \subseteq \bigcup_{j \in J_{2}} G_{j}
$$

whence $S=\bigcup_{j \in J} G_{j}$, and $(\mathcal{S}, \tau, \kappa)$ is nearly compact. Near cocompactness is proved in an analogous way.

To establish near stability let $F \neq S$ have the form $F=\bigcap_{\alpha \in A} F_{\alpha}$ where each $F_{\alpha}$ is regular-closed and let $G_{i}, i \in I$, be regular-open sets with $F \subseteq \bigvee_{i \in I} G_{i}$. Define

$$
\mathcal{C}=\left\{\left(S, F_{\alpha}\right) \mid \alpha \in A\right\} \cup\left\{\left(G_{i}, \emptyset\right) \mid i \in I\right\} .
$$

It is clear that $\mathcal{C}$ is a regular-open, co-regular-closed dicover, and hence has a finite, co-finite sub-dicover $\mathcal{C}_{1}$. Without loss of generality we may assume

$$
\mathcal{C}_{1}=\left\{\left(S, F_{\beta}\right) \mid \beta \in B\right\} \cup\left\{\left(G_{j}, \emptyset\right) \mid j \in J\right\},
$$

where $B \subseteq A$ and $J \subseteq I$ are finite. Now we obtain

$$
F \subseteq \bigcap_{\beta \in B} F_{\beta} \subseteq \bigcup_{j \in J} G_{j}
$$

which shows that $F$ is nearly compact in $S$. Hence $(\tau, \kappa)$ is nearly stable, and near costability can be proved in a similar way.

Hence $(\tau, \kappa)$ is nearly dicompact.

\section{Semibiregulization and the Tychonoff Theorems}

As we have noted before, for any topological space $(X, \mathcal{T})$ the intersection of a finite number of regular-open sets is regular-open . It follows that the regular-open sets in any topological space $(X, \mathcal{T})$ form a base for a topology on $X$. This gives rise to the following definition.

6.1. Definition. The topology generated by the regular-open sets in the topological space $(X, \mathcal{T})$ is called the semiregularization topology of $(X, \mathcal{T})$ and is denoted by $\left(X, \mathcal{T}^{\star}\right)$.

It is clear that $\mathcal{T}^{\star} \subseteq \mathcal{T}$, but the converse is not true in general. Topologies for which $\mathcal{T}=\mathcal{T}^{\star}$ are given a special name.

6.2. Definition. The topological space $(X, \mathcal{T})$ is called semiregular if the regular-open sets form a base for the topology. Equivalently, if $\mathcal{T}^{\star}=\mathcal{T}$.

6.3. Example. For any set $X$, all the open sets in the discrete topology on $X$ and in the indiscrete topology on $X$ are regular-open. Hence these spaces are semiregular. For the usual topology on $\mathbb{R}$ the basic open sets $(a, b)$ are also regular-open, so this topology is semiregular. However the only regular-open sets for the finite complement topology on an infinite set $X$ are $\emptyset$ and $X$, which generate the indiscrete topology on $X$. Hence this space is not semiregular.

In the case of ditopological texture spaces we may define analogous concepts.

6.4. Definition. Let $(S, \mathcal{S}, \tau, \kappa)$ be a ditopological texture space.

(1) Denote by $\tau^{\star}$ the topology generated by the regular-open sets of $(\tau, \kappa)$. Then $\left(\tau^{\star}, \kappa\right)$ is called the semiregularization of $(\tau, \kappa)$. The ditopology $(\tau, \kappa)$ is called semiregular if $\tau^{\star}=\tau$.

(2) Denote by $\kappa^{\star}$ the cotopology generated by the regular-closed sets of $(\tau, \kappa)$. Then $\left(\tau, \kappa^{\star}\right)$ is called the semicoregularization of $(\tau, \kappa)$. The ditopology $(\tau, \kappa)$ is called semicoregular if $\kappa^{\star}=\kappa$. 
(3) The ditopology $\left(\tau^{\star}, \kappa^{\star}\right)$ is called the semibiregularization of $(\tau, \kappa)$ and $(\tau, \kappa)$ is called semibiregular if $\left(\tau^{\star}, \kappa^{\star}\right)=(\tau, \kappa)$.

An important result for topological spaces is that a topological space is nearly compact if and only if the semiregularization topology is compact. For ditopological texture spaces we have the following results.

6.5. Theorem. Let $(\tau, \kappa)$ be a ditopology on $(S \mathcal{S})$.

(1) The following are equivalent:

(a) $(\tau, \kappa)$ is nearly compact.

(b) $\left(\tau^{\star}, \kappa\right)$ is compact.

(c) $\left(\tau^{\star}, \kappa^{\star}\right)$ is compact.

(2) The following are equivalent:

(a) $(\tau, \kappa)$ is nearly cocompact.

(b) $\left(\tau, \kappa^{\star}\right)$ is cocompact.

(c) $\left(\tau^{\star}, \kappa^{\star}\right)$ is cocompact.

Proof. (1) (a) $\Longrightarrow$ (b). Let $\mathcal{C}$ be a cover of $S$ by members of $\tau^{\star}$. Then $\mathcal{C}$ is also a cover of $S$ by members of $\tau$ so there exists a finite subfamily of $\mathcal{C}$, say $C_{i}, i=1 \ldots n$, such that

$$
\left.S=\bigcup_{i=1}^{n}\right]\left[C_{i}\right][.
$$

Since $]\left[C_{i}\right]\left[=C_{i}\right.$ we obtain $S=\bigcup_{i=1}^{n} C_{i}$, so $\left(\tau^{\star}, \kappa\right)$ is compact.

(b) $\Longrightarrow(\mathrm{c})$. Clear since compactness depends only on the topology.

$(\mathrm{c}) \Longrightarrow(\mathrm{a})$. Let $\mathcal{C}$ be a cover of $S$ by member of $\tau$. Then $C \subseteq][C][\forall C \in \mathcal{C}$, where the interior and closure are taken in $(\tau, \kappa)$, and therefore \{]$[C][\mid C \in \mathcal{C}\}$ is a covering of $S$ by members of $\tau^{\star}$. By (c) there exists a finite subfamily of $\mathcal{C}$, say $C_{i}, i=1 \ldots n$, such that

$$
\left.S=\bigcup_{i=1}^{n}\right]\left[C_{i}\right][.
$$

Hence $(\tau, \kappa)$ is nearly compact.

(2). The proof is dual to (1), and is omitted.

We may present the following generalization of Mrówka's characterization of compact topological spaces [13].

6.6. Theorem. Let $\left(S_{1}, \mathcal{S}_{1}, \tau_{1}, \kappa_{1}\right)$ be a ditopological texture space. The following are equivalent:

(1) $\left(S_{1}, \mathcal{S}_{1}, \tau_{1}, \kappa_{1}\right)$ is nearly compact.

(2) For all ditopological spaces $\left(S_{2}, \mathcal{S}_{2}, \tau_{2}, \kappa_{2}\right)$ the projection difunction

$\left(\pi_{2}, \Pi_{2}\right):\left(S_{1}, \mathcal{S}_{1}, \tau_{1}^{*}, \kappa_{1}\right) \times\left(S_{2}, \mathcal{S}_{2}, \tau_{2}, \kappa_{2}\right) \rightarrow\left(S_{2}, \mathcal{S}_{2}, \tau_{2}, \kappa_{2}\right)$

is co-open.

(3) Condition (2) holds for all normal ditopological spaces $\left(S_{2}, \mathcal{S}_{2}, \tau_{2}, \kappa_{2}\right)$.

Proof. In view of Theorem $6.5(1 \mathrm{~b})$ it is sufficient to apply [6, Corollary 2.18] with $\tau_{1}^{*}$ in place of $\tau_{1}$.

Dually,

6.7. Theorem. Let $\left(S_{1}, \mathcal{S}_{1}, \tau_{1}, \kappa_{1}\right)$ be a ditopological texture space. The following are equivalent:

(1) $\left(S_{1}, \mathcal{S}_{1}, \tau_{1}, \kappa_{1}\right)$ is nearly cocompact. 
(2) For all ditopological spaces $\left(S_{2}, \mathcal{S}_{2}, \tau_{2}, \kappa_{2}\right)$ the projection difunction

$\left(\pi_{2}, \Pi_{2}\right):\left(S_{1}, \mathcal{S}_{1}, \tau_{1}, \kappa_{1} *\right) \times\left(S_{2}, \mathcal{S}_{2}, \tau_{2}, \kappa_{2}\right) \rightarrow\left(S_{2}, \mathcal{S}_{2}, \tau_{2}, \kappa_{2}\right)$

is closed.

(3) Condition (2) holds for all normal ditopological spaces $\left(S_{2}, \mathcal{S}_{2}, \tau_{2}, \kappa_{2}\right)$.

6.8. Theorem. Let $(\tau, \kappa)$ be a ditopology on $(S, \mathcal{S})$. Then:

(1) $(\tau, \kappa)$ is nearly stable iff $\left(\tau^{\star}, \kappa^{\star}\right)$ is stable.

(2) $(\tau, \kappa)$ is nearly costable iff $\left(\tau^{\star}, \kappa^{\star}\right)$ is costable.

Proof. (1). Suppose $(\tau, \kappa)$ is nearly stable. Take $F \in \kappa^{\star}$ with $F \neq S$. Then $F=\bigcap K_{\alpha}$, where the $K_{\alpha}$ are regular-closed sets. By near stability $F$ is nearly compact in $(\tau, \kappa)$. However, as in the proof of Theorem 6.5 this means that $F$ is compact in $\left(\tau^{\star}, \kappa^{\star}\right)$. Hence $\left(\tau^{\star}, \kappa^{\star}\right)$ is stable.

The converse is proved is exactly the same way.

(2). This is dual to (1), and the proof is omitted.

6.9. Corollary. The ditopological texture space $(S, \mathcal{S}, \tau, \kappa)$ is nearly dicompact if and only if $\left(S, \mathcal{S}, \tau^{\star}, \kappa^{\star}\right)$ is dicompact.

We recall the following notions from [6, Definition 3.10]:

6.10. Definition. Let $(S, \mathcal{S}, \tau, \kappa)$ be a ditopological texture space and $A \in \mathcal{S}$.

(1) (a) $Q(A)=\bigcap\{] Q_{s}\left[\mid P_{s} \nsubseteq A\right\}$.

(b) $A$ is called pseudo open if $Q(A)=] A[$.

(2) (a) $P(A)=\bigvee\left\{\left[P_{s}\right] \mid A \nsubseteq Q_{s}\right\}$.

(b) $A$ is called pseudo closed if $P(A)=[A]$.

It is shown in [6, Lemma 3.11] that $] A[\subseteq Q(A) \subseteq A$ and $A \subseteq P(A) \subseteq[A]$ for all $A \in \mathcal{S}$. Now we have:

6.11. Theorem. Let $(S, \mathcal{S}, \tau, \kappa)$ be a nearly compact nearly stable ditopological space for which $\left(\tau^{*}, \kappa^{*}\right)$ is $R_{0}$. Then every pseudo closed set in $\left(\tau^{*}, \kappa^{*}\right)$ is nearly compact in $(\tau, \kappa)$.

Proof. By Theorem $6.5(1 \mathrm{c})$ we have that $\left(S, \mathcal{S}, \tau^{*}, \kappa^{*}\right)$ is compact, while by Theorem 6.8 it is stable. Hence by [6, Theorem 3.14(1)] a pseudo closed set $F$ in $\left(S, \mathcal{S}, \tau^{*}, \kappa^{*}\right)$ is compact. It follows as in the proof of Theorem 6.5 that $F$ is nearly compact in $(S, \mathcal{S}, \tau, \kappa)$.

Dually,

6.12. Theorem. Let $(S, \mathcal{S}, \tau, \kappa)$ be a nearly cocompact nearly costable ditopological space for which $\left(\tau^{*}, \kappa^{*}\right)$ is co- $R_{0}$. Then every pseudo open set in $\left(\tau^{*}, \kappa^{*}\right)$ is nearly cocompact in $(\tau, \kappa)$.

In the opposite direction the following is an immediate consequence of $[6$, Theorem 3.15].

6.13. Theorem. Let $(S, \mathcal{S}, \tau, \kappa)$ be a ditopological texture space.

(1) Suppose $\left(\tau^{*}, \kappa^{*}\right)$ is co- $R_{1}$. Then if $A$ is nearly compact in $(\tau, \kappa)$ it is pseudo closed in $\left(\tau^{*}, \kappa^{*}\right)$.

(2) Suppose $\left(\tau^{*}, \kappa^{*}\right)$ is $R_{1}$. Then if $A$ is cocompact in $(\tau, \kappa)$ it is pseudo open in $\left(\tau^{*}, \kappa^{*}\right)$.

Now we may give Tychonoff Theorems for near compactness, near cocompactness and near dicompactness. 
6.14. Theorem. Let $\left(S_{i}, \mathcal{S}_{i}, \tau_{i}, \kappa_{i}\right), i \in I$, be non-empty ditopological texture spaces and $(S, \mathcal{S}, \tau, \kappa)$ their product.

(i) $(S, \mathcal{S}, \tau, \kappa)$ is nearly compact if and only if $\left(S_{i}, \mathcal{S}_{i}, \tau_{i}, \kappa_{i}\right)$ is nearly compact for all $i \in I$.

(ii) $(S, \mathcal{S}, \tau, \kappa)$ is nearly cocompact if and only if $\left(S_{i}, \mathcal{S}_{i}, \tau_{i}, \kappa_{i}\right)$ is nearly cocompact for all $i \in I$.

(iii) $(S, \mathcal{S}, \tau, \kappa)$ is nearly dicompact if and only if $\left(S_{i}, \mathcal{S}_{i}, \tau_{i}, \kappa_{i}\right)$ is nearly dicompact for all $i \in I$.

Proof. We will need the following lemma.

6.15. Lemma. Let $\left(S_{i}, \mathcal{S}_{i}, \tau_{i}, \kappa_{i}\right), i \in I$, be ditopological texture spaces and $(S, \mathcal{S}, \tau, \kappa)$ their product. Let $\left(S, \mathcal{S}, \tau^{\prime}, \kappa^{\prime}\right)$ denote the product of the spaces $\left(S_{i}, \mathcal{S}_{i}, \tau_{i}^{\star}, \kappa_{i}^{\star}\right)$. Then $\left(\tau^{\star}, \kappa^{\star}\right)=\left(\tau^{\prime}, \kappa^{\prime}\right)$.

Proof. The sets $E(i, G), i \in I, G \in \tau_{i}^{\star}$, form a subbase for the topology $\tau^{\prime}$. Since the regular-open sets in $\tau_{i}$ are a base for the topology $\tau_{i}^{\star}$ it follows that the family

$$
\mathcal{G}=\left\{E(i, G) \mid i \in I, G \text { regular-open in } \tau_{i}\right\}
$$

is also a subbase for $\tau^{\prime}$. However $E(i, G)$ is regular-open in $\tau$ if and only if $G$ is regularopen in $\tau_{i}$, so it is not difficult to verify that $\mathcal{G}$ is also a subbase for the topology $\tau^{\star}$. Hence $\tau^{\prime}=\tau^{\star}$, and the proof of $\kappa^{\prime}=\kappa^{\star}$ is dual to this.

We now turn to the proof of the theorem.

(i). Suppose that $(\tau, \kappa)$ is nearly compact. Then, by Theorem $6.5(1 \mathrm{c})$, the ditopology $\left(\tau^{\star}, \kappa^{\star}\right)$ is compact. However, by Lemma $6.15,\left(S, \mathcal{S}, \tau^{\star}, \kappa^{\star}\right)$ is the product of the spaces $\left(S_{i}, \mathcal{S}_{i}, \tau_{i}^{\star}, \kappa_{i}^{\star}\right)$ so by $[6$, Theorem $2.15(\mathrm{i})]\left(S_{i}, \mathcal{S}_{i}, \tau_{i}^{\star}, \kappa_{i}^{\star}\right)$ is compact for all $i \in I$. Hence, by Theorem $6.5(1 \mathrm{c}),\left(S_{i}, \mathcal{S}_{i}, \tau_{i}, \kappa_{i}\right)$ is nearly compact for all $i \in I$.

Conversely, suppose that the spaces $\left(S_{i}, \mathcal{S}_{i}, \tau_{i}, \kappa_{i}\right)$ are nearly compact for all $i \in$ I. Then by Theorem $6.5(1 \mathrm{c})$ the spaces $\left(S_{i}, \mathcal{S}_{i}, \tau_{i}^{\star}, \kappa_{i}^{\star}\right)$ are compact, so by [6, Theorem $2.15(\mathrm{i})],\left(\tau^{\prime}, \kappa^{\prime}\right)=\left(\tau^{\star}, \kappa^{\star}\right)$ is a compact ditopology on $(S, \mathcal{S})$. The product ditopological texture $(S, \mathcal{S}, \tau, \kappa)$ is therefore nearly compact, again by Theorem $6.5(1 \mathrm{c})$.

(ii). The proof is dual to (i), and is omitted.

(iii). Straightforward from (i) and (ii).

We end by recalling an important result from [20]. First we will require the following definition.

6.16. Definition. Let $(S, \mathcal{S})$ be a texture.

(1) $s \in S$ will be called a plain point if $P_{s} \nsubseteq Q_{s}$. The set of plain points of $S$ will be denoted by $S_{p}$.

(2) The texture $(S, \mathcal{S})$ will be called nearly-plain if given $s \in S$ there exists a point in $S_{p}$ with the same q-set as $s$.

It is shown in [20, Proposition 4.1] that every dicompact bi- $T_{2}$ ditopological texture space has a nearly plain texture. This restriction rules out the possibility of a dicompact bi- $T_{2}$ ditopology on, for example, the texture $(L, \mathcal{L})$ of Examples 1.1(1) for this texture has no plain points. Indeed, this texture does not even have the weaker almost plain property discussed in [21]. It is natural to ask if similar restrictions hold for near compactness. This is currently an open question, but the following examples show that there can exist co- $T_{1}$ nearly dicompact ditopologies on such textures. 
6.17. Example. Consider $(L, \mathcal{L})$ with the ditopology $(\tau, \kappa)$ defined by $\tau=\mathcal{L}, \kappa=$ $\{L, \emptyset\}$. As in Examples $2.10(1)$ we see that the only regular-open sets are $L, \emptyset$, whence $\tau^{*}=\kappa^{*}=\{L, \emptyset\}$ and so $(L, \mathcal{L}, \tau, \kappa)$ is nearly dicompact. With regard to separation we see that $Q_{s} \nsubseteq Q_{t} \Longrightarrow t<s$ so we may choose $t<r<s$ and then $G=(0, r] \in \tau$, $P_{s} \nsubseteq G \nsubseteq Q_{t}$, so $(L, \mathcal{L}, \tau, \kappa)$ is co- $T_{1}$ by [5, Theorem 4.11 (2 ii)]. On the other hand by [5, Theorem $4.11(1 \mathrm{i})$ ] it is clear that this space is not $T_{1}$ and hence, in particular not bi- $T_{2}$.

6.18. Example. Consider the Hutton texture of the real texture $(\mathbb{R}, \mathcal{R})$ given in Examples 1.1(4). As shown in [22] this may be represented by $\left(M_{\mathbb{R}}, \mathcal{M}_{\mathbb{R}}\right)$ where $M_{\mathbb{R}}=$ $(\mathbb{R} \times\{0\}) \cup(\mathbb{R} \times\{1\}) \cup\{\infty\}$ and $\mathcal{M}_{\mathbb{R}}=\left\{U_{r} \mid r \in \mathbb{R}\right\} \cup\left\{V_{r} \mid r \in \mathbb{R}\right\} \cup\left\{M_{\mathbb{R}}\right\}$, where

$$
U_{r}=(-\infty, r] \times\{0,1\}, V_{r}=(-\infty, r) \times\{0\} \cup(-\infty, r] \times\{1\} .
$$

Moreover, $P_{(r, 0)}=U_{r}, Q_{(r, 0)}=V_{r} ; P_{(r, 1)}=Q_{(r, 1)}=V_{r} ; P_{\infty}=Q_{\infty}=M_{\mathbb{R}}$. As noted in [22] this texture is not nearly plain since $\infty$ is a non-plain point whose q-set is not equal to the q-set of any of the plain points. If we take $\tau=\left\{V_{r} \mid r \in \mathbb{R}\right\} \cup\left\{M_{\mathbb{R}}, \emptyset\right\}, \kappa=\left\{M_{\mathbb{R}}, \emptyset\right\}$ then much as in the above example we see that $\tau^{*}=\kappa^{*}=\left\{M_{\mathbb{R}}, \emptyset\right\}$, whence $\left(M_{\mathbb{R}}, \mathcal{M}_{\mathbb{R}}, \tau, \kappa\right)$ is nearly dicompact. Again, this space is co- $T_{1}$. To see this using [5, Theorem 4.11 (2 ii)] note that we have $Q_{\infty} \nsubseteq Q_{(s, k)}$ for any $s \in \mathbb{R}, k=0,1$, and $Q_{(s, m)}=V_{s} \nsubseteq V_{t}=Q_{(t, n)}$ for $t<s$ and $m, n=0,1$. In the first case we may take $r>s, G=V_{r} \in \tau$ to give

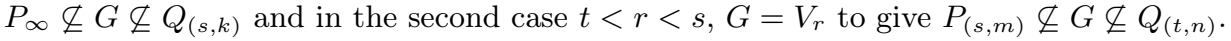

As in the above example this space also is not $T_{1}$ and hence in particular not bi- $T_{2}$.

\section{Acknowledgement}

The authors thank the referees for their helpful comments

\section{References}

[1] Brown, L. M. and Diker, M. Ditopological texture spaces and intuitionistic sets, Fuzzy Sets and Systems 98, 217-224, 1998.

[2] Brown, L. M. and Ertürk, R. Fuzzy Sets as Texture Spaces, I. Representation Theorems, Fuzzy Sets and Systems 110 (2), 227-236, 2000.

[3] Brown, L. M., Ertürk, R. and Dost, Ş. Ditopological texture spaces and fuzzy topology, I. Basic Concepts, Fuzzy Sets and Systems 147 (2), 171-199, 2004.

[4] Brown, L. M., Ertürk, R. and Dost, Ş. Ditopological texture spaces and fuzzy topology, II. Topological Considerations, Fuzzy Sets and Systems 147 (2), 201-231, 204.

[5] Brown, L. M., Ertürk, R. and Dost, Ş. Ditopological texture spaces and fuzzy topology, III. Separation Axioms, Fuzzy Sets and Systems 157 (14), 1886-1912, 2006.

[6] Brown, L. M. and Gohar, M. M. Compactness in ditopological texture spaces, Hacet. J. Math. Stat. 38 (1), 21-43, 2009.

[7] Carnahan, D. Some properties related to compactness in topological spaces (PhD Dissertation, University of Arkansas, 1973).

[8] Gierz, G., Hofmann, K. H., Keimel, D., Lawson, J., Mislove, M. and Scott, D. A compendium of continuous lattices (Springer-Verlag, Berlin, Heidelberg, New York, 1980).

[9] Gohar, M. M. Compactness in ditopological texture spaces (PhD Thesis, Hacettepe University, 2002).

[10] Gohar, M. M. and Brown, L. M. Strong compactness of ditopological texture spaces, AIP Conference Proceedings 1309, 153-168, 2010.

[11] Herrington, L. L. Properties of nearly-compact spaces, Proc. Amer. Math. Soc. 45, 431-436, 1974.

[12] Kopperman, R. D. Asymmetry and duality in topology, Topology and its Applications 66, 1-39, 1995.

[13] Mrówka, S. Compactness and product spaces, Coll.Math 7, 19-22, 1959.

[14] Özçă̆, S. and Brown, L. M. Di-uniform texture spaces, Appl. Gen. Top. 4 (1), 157-192, 2003. 
[15] Özçă̆, S., Brown, L.M. and Krsteska, B. Di-uniformities and Hutton uniformities, Fuzzy Sets and Systems 195, 58-74, 2012.

[16] Singal, M. K. and Mathur, A. On nearly compact spaces, Boll Un. Mat. Ital. 6 (4), 702-710, 1969.

[17] Singal, M. K. and Mathur, A. On nearly compact spaces (II), Boll. Un. Mat. Ital. 9 (4), 1974.

[18] Tiryaki, İ. U. Fuzzy sets over the poset $\mathbb{I}$, Hacet. J. Math. Stat. 37 (2), 143-166, 2008.

[19] Yıldız, F. and Brown, L.M. Categories of dicompact bi-T $T_{2}$ texture spaces and a BanachStone theorem, Quaestions Mathematicae 30, 167-192, 2007.

[20] Yıldiz, F. and Brown, L. M. Real dicompact textures, Topology and its Applications 156 (11), 1970-1984, 2009.

[21] F. Yıldız and Brown, L. M. Real dicompactifications of ditopological texture spaces, Topology and its Applications 156 (18), 3041-3051, 2009.

[22] Ylldiz, F. and Brown, L. M. Extended real dicompactness and an application to Hutton spaces, Fuzzy Sets and Systems 227, 74-95, 2013.

[23] Yıldız, G. and Ertürk, R. Di-extremities on textures, Hacet. J. Math. Stat. 38 (3), 243-257, 2009. 